\title{
Histopathologic heterogeneity of acute respiratory distress syndrome revealed by surgical lung biopsy and its clinical implications
}

Jimyung Park ${ }^{1}$, Yeon Joo Lee ${ }^{2}$, Jinwoo Lee ${ }^{1}$, Sung Soo Park ${ }^{3}$, Young-Jae Cho ${ }^{2}$, Sang-Min Lee ${ }^{1}$, Young Whan Kim ${ }^{1}$, Sung Koo Han ${ }^{1}$, and Chul-Gyu Yoo ${ }^{1}$

\begin{abstract}
${ }^{1}$ Division of Pulmonary and Critical Care Medicine, Department of Internal Medicine, Seoul National University Hospital, Seoul; ${ }^{2}$ Division of Pulmonary and Critical Care Medicine, Department of Internal Medicine, Seoul National University Bundang Hospital, Seongnam; ${ }^{3}$ Division of Pulmonary and Critical Care Medicine, Department of Internal Medicine, Seoul Metropolitan Government Seoul National University Boramae Medical Center, Seoul, Korea
\end{abstract}

Received: October 29, 2016 Revised : March 19, 2017 Accepted: May 16, 2017

\section{Correspondence to}

Chul-Gyu Yoo, M.D.

Division of Pulmonary and

Critical Care Medicine,

Department of Internal Medicine,

Seoul National University

Hospital, 101 Daehak-ro,

Jongno-gu, Seoul 03080, Korea

Tel: $+82-2-2072-3760$

Fax: +82-2-2072-0630

E-mail: cgyoo@snu.ac.kr
Background/Aims: Diffuse alveolar damage (DAD) is the histopathologic hallmark of acute respiratory distress syndrome (ARDS). However, there are several non-DAD conditions mimicking ARDS. The purpose of this study was to investigate the histopathologic heterogeneity of ARDS revealed by surgical lung biopsy and its clinical relevance.

Methods: We retrospectively analyzed 84 patients with ARDS who met the criteria of the Berlin definition and underwent surgical lung biopsy between January 2004 and December 2013 in three academic hospitals in Korea. We evaluated their histopathologic findings and compared the clinical outcomes. Additionally, the impact of surgical lung biopsy on therapeutic alterations was examined.

Results: The histopathologic findings were highly heterogeneous. Of 84 patients undergoing surgical lung biopsy, DAD was observed in 31 patients (36.9\%), while 53 patients (63.1\%) did not have DAD. Among the non-DAD patients, diffuse interstitial lung diseases and infections were the most frequent histopathologic findings in 19 and 17 patients, respectively. Although the mortality rate was slightly higher in DAD (71.0\%) than in non-DAD (62.3\%), the difference was not significant. Overall, the biopsy results led to treatment alterations in 40 patients (47.6\%). Patients with non-DAD were more likely to change the treatment than those with DAD (58.5\% vs. $29.0 \%)$, but there were no significant improvements regarding the mortality rate.

Conclusions: The histopathologic findings of ARDS were highly heterogeneous and classic DAD was observed in one third of the patients who underwent surgical lung biopsy. Although therapeutic alterations were more common in patients with non-DAD-ARDS, there were no significant improvements in the mortality rate.

Keywords: Acute lung injury; Biopsy; Pathology; Respiratory distress syndrome, adult

\section{INTRODUCTION}

Acute respiratory distress syndrome (ARDS) is an acute hypoxemic respiratory failure with diffuse pulmonary infiltrates caused by lung injury due to various underlying conditions [1]. A diagnosis of ARDS is currently 
established based on the clinical criteria of the Berlin definition, which were proposed in 2012 [2]. Upon histopathologic examination, ARDS typically presents with diffuse alveolar damage (DAD), which is the final histopathologic feature and a reaction of the lung to various injuries [3]. However, the presence of DAD on histopathology is not mandatory for the diagnosis because ARDS is defined clinically. Several non-DAD histopathologic conditions can mimic the clinical manifestations of ARDS [4]. Therefore, ARDS can be divided into two groups based on histopathology: DAD-ARDS and non-DAD-ARDS.

Currently, there are no proven pharmacologic therapies for DAD-ARDS [5]. In contrast, specific pharmacologic and therapeutic options may be available for patients with non-DAD-ARDS (i.e., specific antimicrobial therapy for pneumonia). Therefore, after the reliable and accurate diagnosis of patients with non-DAD-ARDS, a prompt initiation of specific treatment may allow for an improved prognosis. In a clinical setting, however, differentiation between DAD-ARDS and non-DAD-ARDS is difficult. Non-invasive diagnostic methods, such as computed tomography and bronchoscopy, are not effective for this differential diagnosis. Further, there are no known biomarkers for DAD [6]. Therefore, the only approach to diagnose $\mathrm{DAD}$ is via histopathologic examination of the lung tissue obtained by surgical lung biopsy.

Histopathologic examination has indicated that a variety of non-DAD conditions clinically manifest as ARDS. Additionally, the frequency of non-DAD-ARDS has been reported to be substantial on surgical lung biopsy [7-14]. Postmortem studies have also demonstrated that the finding of DAD could only be confirmed in approximately half of the patients with clinical ARDS $[15,16]$. Recently, several studies evaluating the differences between the clinical manifestation and prognosis of DAD-ARDS and non-DAD-ARDS have provided insight into the heterogeneity of ARDS [17-19]. However, in Korea, there is a lack of data concerning the frequency of non-DAD histopathologic conditions of ARDS. Therefore, the purpose of this study was to evaluate the histopathologic heterogeneity of ARDS and its clinical implications in patients in Korea.

\section{METHODS}

\section{Study population}

This was a retrospective cohort study conducted in the intensive care units (ICUs) of three academic hospitals in Korea (Seoul National University Hospital, Seoul National University Bundang Hospital, and Seoul Metropolitan Government-Seoul National University Boramae Medical Center). The Institutional Review Board of each institution approved this study prior to the data collection. We included all patients with ARDS who fulfilled the criteria of the Berlin definition and who underwent surgical lung biopsy between January 2004 and December 2013. Using the Berlin definition, ARDS was categorized as mild if the partial pressure of arterial oxygen $\left(\mathrm{PaO}_{2}\right)$ /fraction of inspired oxygen $\left(\mathrm{FiO}_{2}\right)$ ratio was between 201 and $300 \mathrm{mmHg}$, moderate if it was between 101 and $200 \mathrm{mmHg}$, and severe if it was $100 \mathrm{mmHg}$ or less [2]. The severity of ARDS was determined by the results of the arterial blood gas analysis on the date of the lung biopsy. Patients were also classified as either immunocompromised or immunocompetent, depending on their comorbidities. Immunocompromised patients were those who previously underwent organ transplantation, or who had malignant diseases, hematologic diseases, connective tissue diseases, or human immunodeficiency virus infection.

\section{Surgical lung biopsy}

Surgical lung biopsy was considered in patients with ARDS of unknown etiology after intensive evaluation, including imaging and bronchoscopy, and the decision whether to perform a biopsy was made by the attending physicians. As patients were mechanically ventilated and unable to communicate, their surrogates provided informed consent before the surgical lung biopsy. The biopsy procedure was performed in the ICU or the operating room by experienced thoracic surgeons. Patients not eligible for transport to the operating room underwent the biopsy procedure at the bedside in the ICU. The choice of surgical method between open lung biopsy via thoracotomy and video-assisted thoracoscopic surgery (VATS), and the decision regarding which lobe to resect were discussed between the thoracic surgeons and the attending physicians. Patients deemed unable to tolerate a one-lung ventilation procedure underwent 
open lung biopsy. Otherwise, VATS lung biopsy was attempted.

\section{Histopathologic examination}

The lung tissue obtained by surgical lung biopsy was reviewed by the pathologists at each institution. In addition to routine hematoxylin and eosin staining, other staining methods, including Gomori methenamine silver and periodic acid-Schiff staining, specific immunohistochemistry, or polymerase chain reaction assays were performed as necessary. If infection was suspected, some of the biopsied tissue was used for microbiologic cultures.

The histopathologic results of the lung biopsy were divided into two categories: DAD and non-DAD. DAD was diagnosed if hyaline membrane formation was evident and at least one of the following was observed: edema, necrosis, proliferation, or fibrosis [3]. Non-DAD histopathologic results included diffuse interstitial lung diseases, infections, and other uncommon conditions. Infections were diagnosed if specific microorganisms were identified by histopathology.

\section{Clinical data collection}

The following information was collected: age, sex, comorbidities, dates of hospital and ICU admission, Acute Physiology and Chronic Health Evaluation II (APACHE II) scores on ICU admission, results of other diagnostic evaluations performed before biopsy, dates of the surgical lung biopsy, details of biopsy procedures and results, treatment alterations made based on biopsy results, procedure-related complications, dates of ICU and hospital discharge, and in-hospital mortality.

Treatment alterations were defined as the initiation or discontinuation of drugs such as antimicrobial agents, corticosteroids, immunosuppressants, or the introduction of a new therapeutic procedure (i.e., plasmapheresis). Empirical treatment alterations that had begun prior to the report of biopsy results were not considered as treatments prescribed as a consequence of the biopsy results. Procedure-related complications were classified as either major complications or minor complications. Mortality, clinically significant hemorrhage, and reoperation for any reason after biopsy were considered major complications. The list of minor complications, on the other hand, comprised persistent air leaks, pneu- mothorax requiring additional chest tube insertion, and wound infection.

\section{Statistical analysis}

Data were reported as means with standard deviations for continuous variables with normal distribution, or medians with interquartile range (IQR) for those with non-normal distribution. For categorical variables, values were reported as frequencies and proportions. Differences in categorical variables between the subgroups were analyzed using either the chi-square test or Fisher exact test. Continuous variables were analyzed by Student $t$ test or Mann-Whitney $U$ test. A $p$ value less than 0.05 was considered statistically significant in a two-tailed test. Statistical analyses were performed using STATA version 14.0 (StataCorp, College Station, TX, USA).

\section{RESULTS}

\section{Patient characteristics and biopsy procedure}

A total of 84 patients with ARDS who underwent surgical lung biopsy between January 2004 and December 2013 were analyzed. The baseline demographic characteristics and the severity of ARDS at the time of the biopsy are shown in Table 1 . Their median age was 62 years (IQR, 49 to 71 ) and the median $\mathrm{PaO}_{2} / \mathrm{FiO}_{2}$ ratio at the time of the biopsy was $123.3 \mathrm{mmHg}$ (IQR, 91.2 to 165.7). Among the 84 patients, 29 patients (34.5\%) had severe ARDS, 45 patients (53.6\%) had moderate ARDS, and the remaining ten patients (11.9\%) had mild ARDS, according to the Berlin definition [2]. Thirty-eight patients (45.2\%) were classified as immunocompromised due to various conditions (Table 2). The most common etiology of immunocompromised patients was connective tissue disease requiring immunosuppressants, followed by chemotherapy for malignant diseases.

Of the 84 patients, 43 patients (51.2\%) underwent open lung biopsy via thoracotomy and 41 patients (48.8\%) underwent VATS lung biopsy. Patients who underwent VATS lung biopsy had a higher median $\mathrm{PaO}_{2} / \mathrm{FiO}_{2}$ ratio compared to those who underwent open lung biopsy (139.3 mmHg vs. $98.5 \mathrm{mmHg}, p=0.001$ ). The median duration from ICU admission to lung biopsy was 2 days, and 71 patients (84.5\%) underwent the biopsy procedure 
within 1 week after ICU admission.

\section{Heterogeneity of histopathologic results}

Histopathologic examination revealed highly heterogeneous results (Table 3). DAD was observed in 31 patients (36.9\%), while non-DAD was observed in 53 patients (63.1\%). When comparing the DAD-ARDS and nonDAD-ARDS groups, no differences were found between the demographic characteristics and the severity of ARDS, as determined by the $\mathrm{PaO}_{2} / \mathrm{FiO}_{2}$ ratio. In patients with DAD-ARDS, the median $\mathrm{PaO}_{2} / \mathrm{FiO}_{2}$ ratio was 140.0 $\mathrm{mmHg}$ and in those with non-DAD-ARDS, the median $\mathrm{PaO}_{2} / \mathrm{FiO}_{2}$ ratio was $112.1 \mathrm{mmHg}(p=0.502)$. The proportion of immunocompromised patients tended to be higher among patients with non-DAD-ARDS compared to those with DAD-ARDS, but this difference was not statistically significant ( $50.9 \%$ vs. $35.5 \%, p=0.170)$.

Among the 53 patients with non-DAD-ARDS, diffuse interstitial lung diseases and infections were most com-

Table 1. Baseline characteristics of 84 patients with ARDS who underwent surgical lung biopsy

\begin{tabular}{lc}
\hline Characteristic & Value \\
\hline Age, yr & $62(49-71)$ \\
Male sex & $51(60.7)$ \\
$\mathrm{PaO}_{2} / \mathrm{FiO}_{2}, \mathrm{mmHg}^{\mathrm{a}}$ & $123.3(91.8-164.4)$ \\
$\mathrm{ARDS}$ severity & \\
Mild & $29(34.5)$ \\
Moderate & $45(53.6)$ \\
Severe & $10(11.9)$ \\
APACHE II score & $23.7 \pm 9.1$ \\
Biopsy method & $43(51.2)$ \\
\hline Open biopsy & $41(48.8)$ \\
\hline VATS biopsy & $2(0-5)$ \\
Days from ICU admission to biopsy & \\
Immune status & $46(54.8)$ \\
\hline Immunocompetent & $38(45.2)$ \\
\hline Immunocompromised
\end{tabular}

Values are presented as median (interquartile range), number $(\%)$, or mean $\pm \mathrm{SD}$.

ARDS, acute respiratory distress syndrome; $\mathrm{PaO}_{2}$, partial pressure of arterial oxygen; $\mathrm{FiO}_{2}$, fraction of inspired oxygen; APACHE II, Acute Physiology and Chronic Health Evaluation II; VATS, video-assisted thoracic surgery; ICU, intensive care unit.

${ }^{a} A R D S$ severity was determined according to the Berlin definition. monly diagnosed in 19 and 17 patients, respectively. Most of the 19 patients diagnosed with diffuse interstitial lung diseases, including organizing pneumonia, usual interstitial pneumonia (UIP), and nonspecific interstitial pneumonia (NSIP), did not show the typical radiological findings. Therefore, it was difficult to establish a diagnosis without histopathology. Seventeen patients were diagnosed with infectious etiologies: Pneumocystis jiroveci pneumonia $(\mathrm{n}=8)$, cytomegalovirus $(\mathrm{CMV})$ pneumonia $(n=4)$, invasive pulmonary aspergillosis $(n=3)$, and pulmonary tuberculosis $(n=2)$. Other than interstitial lung diseases and infections, some patients were diagnosed with vasculitis, primary pulmonary lymphoma, or graft versus host disease. In 10 patients, the histopathologic findings were inconclusive.

\section{Treatment alteration based on lung biopsy results}

After surgical lung biopsy, 40 patients (47.6\%) had therapeutic alterations based on their histopathologic results (Table 4). In these 40 patients, the most common change in their therapeutic regimen involved the initiation

Table 2. The underlying conditions of 38 immunocompromised patients

\begin{tabular}{|cc}
\hline Cause of immunocompromised state & Frequency (\%) \\
\hline Connective tissue disease & $13(34.2)$ \\
\hline Rheumatoid arthritis & 3 \\
\hline Systemic lupus erythematosus & 3 \\
\hline Dermatomyositis & 2 \\
\hline Churg-Strauss syndrome & 1 \\
\hline Polyarteritis nodosa & 1 \\
\hline Microscopic polyangiitis & 1 \\
\hline Spondyloarthropathy & 1 \\
\hline Polymyalgia rheumatica & 1 \\
\hline Malignancy & $11(28.9)$ \\
\hline Lymphoma & 5 \\
\hline Lung cancer & 4 \\
\hline Leukemia & 2 \\
\hline Organ transplantation & $6(15.8)$ \\
\hline Kidney transplantation & 5 \\
\hline Liver transplantation & 1 \\
\hline Hematologic disease & $5(13.2)$ \\
\hline Myelodysplastic syndrome & 4 \\
\hline Aplastic anemia & 1 \\
\hline Human immunodeficiency virus infection & $3(7.9)$ \\
\hline
\end{tabular}


or withdrawal of antimicrobial agents. In 21 patients, unnecessary empirical antimicrobial agents were discontinued and in eight patients, specific antimicrobial agents were prescribed. Patients with non-DAD-ARDS were significantly more likely to change their treatment compared to those with DAD-ARDS (58.5\% vs. $29.0 \%, p$ $=0.009)$. Particularly, in 17 patients who showed infectious etiologies on histopathology, 13 patients (76.5\%) were prescribed more optimal antimicrobial therapies. In addition, corticosteroids or other immunosuppressive agents were prescribed to seven patients diagnosed with NSIP, organizing pneumonia, or graft versus host disease. Two patients diagnosed with primary pulmonary lymphoma were initiated on chemotherapy, and another two patients diagnosed with vasculitis subsequently underwent plasmapheresis.

\section{Clinical outcomes}

Of the 84 patients included in this study, 55 patients (65.5\%) died. The mortality rate was slightly higher in patients with DAD-ARDS (22 out of 31, 71.0\%) than in those with non-DAD-ARDS (33 out of 53, 62.3\%); however, this difference was not statistically significant $(p=$

Table 3. Histopathologic results of surgical lung biopsy

\begin{tabular}{|cc}
\hline Histopathologic result & Frequency $(\%)$ \\
\hline Diffuse alveolar damage & $31(36.9)$ \\
\hline Non-diffuse alveolar damage & $53(63.1)$ \\
\hline Diffuse interstitial lung disease & $19(22.7)$ \\
\hline Organizing pneumonia & 8 \\
\hline Usual interstitial pneumonia & 7 \\
\hline Nonspecific interstitial pneumonia & 4 \\
\hline Infection & $17(20.2)$ \\
\hline Pneumocystis jiroveci pneumonia & 8 \\
\hline Cytomegalovirus pneumonia & 4 \\
\hline Invasive aspergillosis & 2 \\
\hline Pulmonary tuberculosis & $17(20.2)$ \\
\hline Others & 3 \\
\hline Pulmonary vasculitis & 2 \\
\hline Primary pulmonary lymphoma & 1 \\
\hline Alveolar proteinosis & 1 \\
\hline Graft versus host disease & 10 \\
\hline Inconclusive ${ }^{\text {a }}$ & \\
\hline Including histopathologic results that were not distin- \\
guished as a specific disease entity, such as chronic inflam- \\
mation and fibrosis.
\end{tabular}

0.418). Among the patients with non-DAD-ARDS, 11 out of 19 patients $(57.9 \%)$ with diffuse interstitial lung diseases and 10 out of 17 patients (58.8\%) with infections died.

Although therapeutic alterations occurred more frequently in patients with non-DAD-ARDS, the mortality rate did not significantly differ between patients who changed their treatment after biopsy and those who did not $(57.5 \%$ vs. $72.7 \%, p=0.143)$. Patients who had therapeutic alterations to their course of antimicrobial agents had a mortality rate of $55.2 \%$ ( 16 out of 29 ). Of the seven patients who received corticosteroids or immunosuppressants after biopsy, four patients (57.1\%) died.

\section{Procedure-related complications}

After surgical lung biopsy, procedure-related complications occurred in 18 patients (21.4\%). Most of the complications were minor, such as a prolonged air leak or the development of pneumothorax. However, two patients underwent reoperation to control postoperative

Table 4. Treatment alterations based on the histopathologic results

\begin{tabular}{|c|c|}
\hline Treatment alteration & Frequency (\%) \\
\hline Diffuse alveolar damage & 31 \\
\hline Withdrawal of antimicrobial agent & $9(29.0)$ \\
\hline No change & $22(71.0)$ \\
\hline Diffuse interstitial lung disease & 19 \\
\hline Immunosuppressant & $6(31.6)$ \\
\hline Withdrawal of antimicrobial agent & $3(15.8)$ \\
\hline No change & $10(52.6)$ \\
\hline Infection & 17 \\
\hline Initiation of antimicrobial agent & $8(47 \cdot 1)$ \\
\hline Withdrawal of antimicrobial agent & $5(29.4)$ \\
\hline No change & $4(23 \cdot 5)$ \\
\hline Others & 17 \\
\hline Withdrawal of antimicrobial agent & $4(23.5)$ \\
\hline Plasmapheresis $^{\mathrm{a}}$ & $2(11.8)$ \\
\hline Chemotherapy $^{\mathrm{a}}$ & $2(11.8)$ \\
\hline Immunosuppressant $^{\mathrm{a}}$ & $1(5.8)$ \\
\hline No change & $8(47.1)$ \\
\hline
\end{tabular}

${ }^{\mathrm{a}}$ Plasmapheresis was performed for two patients who were diagnosed with vasculitis, and two patients who were diagnosed with primary pulmonary lymphoma received chemotherapy. One patient was started on immunosuppressant therapy for graft versus host disease. 
bleeding. There were no acute procedure-related mortalities. No differences were found in the complication rates between patients who underwent VATS lung biopsy and those who underwent open lung biopsy (19.5\% vs. $23.3 \%, p=0.676$ ).

\section{DISCUSSION}

ARDS is a clinical syndrome with respiratory failure caused by non-cardiogenic pulmonary edema, which results from a variety of insults to the lung. It was first described in 1967 as a collection of 12 cases of acute respiratory failure [20]. Although there has been dramatic progress in ICU care, ARDS is still a common indication for ICU admission and it remains a major cause of ICU mortality [21]. To improve the clinical recognition of ARDS, the diagnostic criteria comprising clinical and radiological findings were developed at the consensus conference of American and European investigators in 1994 [22]. The diagnostic criteria were subsequently revised as the Berlin definition in 2012 [2].

Since its description in the 1970s, ARDS has been known to typically present with DAD upon histopathologic examination [23]. However, as the diagnostic criteria for ARDS include clinical and radiological findings rather than histopathologic findings, numerous nonDAD conditions may fulfill the diagnostic criteria for ARDS on a clinical basis. Previous postmortem studies have shown that DAD was present in only half of the patients with ARDS $[15,16]$. As non-DAD-ARDS conditions may be treated with distinct therapeutic strategies, there have been efforts to differentiate DAD-ARDS from nonDAD-ARDS $[24,25]$. However, the differential diagnosis of the two conditions necessitates histopathologic examination via surgical lung biopsy, as there are currently no known specific biomarkers for DAD.

In the present study, 84 patients with ARDS underwent surgical lung biopsy to reveal the underlying conditions of ARDS, and the non-DAD histopathologic findings were observed in $63.1 \%$ of the patients. In previous studies, the frequency of non-DAD histopathologic findings in clinically diagnosed patients with ARDS varied widely, from $40 \%$ to $70 \%$ [7-14]. Selection biases might have been inevitable as most of the studies on patients with ARDS undergoing surgical lung biopsy, in- cluding our study, were retrospective cohort studies. To illustrate, atypical patients with ARDS who lack a definite precipitating cause are more often considered for surgical lung biopsy than are typical patients with ARDS who have a definite precipitating cause. This particular selection bias may be responsible for the high frequency of non-DAD histopathologic findings. The considerable proportion of immunocompromised patients also reflects this selection bias.

Compared to the patients with classic DAD-ARDS, those with non-DAD-ARDS have been reported to exhibit more favorable oxygenation and lung mechanics and have lower mortality rates [17-19]. In our study, although the mortality rate was slightly higher in patients with DAD-ARDS (71.0\%) than in those with non-DADARDS (62.3\%), the difference was not statistically significant. There were also no significant differences in the $\mathrm{PaO}_{2} / \mathrm{FiO}_{2}$ ratio between patients with DAD-ARDS and those with non-DAD-ARDS. It is widely accepted that ARDS is a heterogeneous syndrome. However, no consensus exists on how to subdivide ARDS into subphenotypes until now [26]. Further studies are needed to elucidate whether it is clinically relevant to divide ARDS into DAD-ARDS and non-DAD-ARDS based on histopathologic exam.

In this study, approximately half of the patients had therapeutic alterations based on the results of the surgical lung biopsy. This proportion was slightly lower than that reported previously. The recent meta-analyses on surgical lung biopsy in ARDS reported that therapeutic changes occurred in about $80 \%$ of patients after biopsy $[27,28]$. This difference may have occurred because we excluded the consideration of corticosteroid therapy as a specific therapeutic alteration for DAD. We justified this decision as the evidence regarding the efficacy of corticosteroid therapy in DAD is inconclusive yet. Among the treatment alterations, the most common alteration was antibiotic de-escalation. As infection is the most common precipitating cause of ARDS, and because the clinical findings of ARDS mimic severe infection, patients with ARDS are usually treated with multiple antimicrobial agents empirically. However, it is well-known that the excessive use of broad-spectrum antimicrobial agents is responsible for several adverse drug events and the emergence of multidrug resistant microorganisms in ICUs [29]. In this regard, surgical lung biopsy may 
contribute to antimicrobial stewardship. However, the efficacy of de-escalation strategies in improving individual patient outcomes remains controversial [30]. This may explain why therapeutic alterations, based on surgical lung biopsy results, failed to improve mortality rate in this study.

Although this study showed that surgical lung biopsy could reveal various non-DAD histopathologic conditions, its clinical role in the diagnosis and treatment of patients with ARDS is not definitive. First, the clinical utility of lung biopsy for the detection of atypical infectious etiologies is being challenged because of the significant improvements in microbiological diagnostic techniques. Bronchoscopy and bronchoalveolar lavage, which are less invasive procedures compared to surgical lung biopsy, are very effective in identifying the infectious etiological agent of diffuse lung infiltrates, including atypical pathogens, such as P.jiroveci, CMV, and Aspergillus [31-34]. Considering the non-negligible complication rates of the surgical lung biopsy in patients with ARDS, physicians should consider such alternative methods before proceeding to surgery [28]. Second, it is unclear whether defining underlying interstitial lung diseases is helpful in the treatment of acute exacerbations presenting as ARDS because corticosteroid is the mainstay of treatment in such setting, regardless of underlying histopathology [35,36]. Although new pharmacologic options for UIP have been recently developed, extensive studies on these drugs in critically ill patients on mechanical ventilation have not been conducted $[37,38]$.

There are critics who consider defining the underlying histopathology of ARDS to be largely an academic exercise. Ideally, a well-designed prospective study, comparing patients with ARDS who undergo surgical lung biopsy with those who do not, is necessary for a more conclusive evaluation of the benefits of the procedure. To the best of our knowledge, no such studies have been conducted. Therefore, the recommendation of surgical lung biopsy should only be made on a caseby-case basis, and a one-size-fits-all invasive approach cannot be recommended for patients with ARDS.

The present study has several differences compared to previous studies in evaluating the use of surgical lung biopsy in patients with ARDS. For instance, the sample size was substantially larger than that of previous stud- ies. Additionally, surgical lung biopsy was performed very early on the course of ARDS (median 2 days from ICU admission). VATS biopsy was also performed in a considerable number of patients. Furthermore, this was the first study to address the various histopathologic findings of ARDS in Korea.

However, there were also limitations to be noted. First, due to the retrospective study design, we could not fully address whether the performance of surgical lung biopsy improved the patient outcomes, such as the mortality rate, length of hospitalization, or ventilator-free days. Second, only a very small proportion of patients with ARDS underwent lung biopsy, making it difficult to generalize the findings of this study. The decision to perform lung biopsy was not made at random. This selection bias should be considered when interpreting the high frequency of non-DAD-ARDS. Third, the predisposing factors to ARDS, such as pneumonia, sepsis, or trauma could not be evaluated due to the retrospective design.

In conclusion, in Korea, surgical lung biopsy in patients with ARDS revealed highly heterogeneous histopathologic results. There was no significant difference in the mortality rates between patients with non-DADARDS and those with DAD-ARDS. Therapeutic alterations were made in approximately half of the patients, and patients with non-DAD-ARDS were more likely to change their treatment. However, a change in treatment did not result in a significant improvement in the mortality rate. Therefore, although surgical lung biopsy can be an option for patients with ARDS for the identification of unexpected underlying conditions, the decision to perform this procedure should be individualized.

\section{KEY MESSAGE}

1. Surgical lung biopsy in patients with acute respiratory distress syndrome (ARDS) revealed significant heterogeneity in the histopathology of the condition. Overall, non-diffuse alveolar damage (DAD)-ARDS was more common than classic DAD-ARDS.

2. Patients with non-DAD-ARDS were more likely to have therapeutic alterations based on histopathologic results. However, this did not lead to a significant improvement in the mortality rate. 


\section{Conflicts of interest}

No potential conflict of interest relevant to this article was reported.

\section{REFERENCES}

1. Ware LB, Matthay MA. The acute respiratory distress syndrome. N Engl J Med 2000;342:1334-1349.

2. ARDS Definition Task Force, Ranieri VM, Rubenfeld GD, et al. Acute respiratory distress syndrome: the Berlin Definition. JAMA 2012;307:2526-2533.

3. Tomashefski JF Jr. Pulmonary pathology of acute respiratory distress syndrome. Clin Chest Med 2000;21:435-466.

4. Schwarz MI, Albert RK. "Imitators" of the ARDS: implications for diagnosis and treatment. Chest 2004;125:15301535 .

5. Spieth PM, Zhang H. Pharmacological therapies for acute respiratory distress syndrome. Curr Opin Crit Care 2014;20:113-121.

6. Bhargava M, Wendt $\mathrm{CH}$. Biomarkers in acute lung injury. Transl Res 2012;159:205-217.

7. Baumann HJ, Kluge S, Balke L, et al. Yield and safety of bedside open lung biopsy in mechanically ventilated patients with acute lung injury or acute respiratory distress syndrome. Surgery 2008;143:426-433.

8. Charbonney E, Robert J, Pache JC, Chevrolet JC, Eggimann P. Impact of bedside open lung biopsies on the management of mechanically ventilated immunocompromised patients with acute respiratory distress syndrome of unknown etiology. J Crit Care 2009;24:122-128.

9. Chuang ML, Lin IF, Tsai YH, Vintch JR, Pang LC. The utility of open lung biopsy in patients with diffuse pulmonary infiltrates as related to respiratory distress, its impact on decision making by urgent intervention, and the diagnostic accuracy based on the biopsy location. J Intensive Care Med 2003;18:21-28.

10. Guerin C, Bayle F, Leray V, et al. Open lung biopsy in nonresolving ARDS frequently identifies diffuse alveolar damage regardless of the severity stage and may have implications for patient management. Intensive Care Med 2015;41:222-230.

11. Kao KC, Tsai YH, Wu YK, et al. Open lung biopsy in early-stage acute respiratory distress syndrome. Crit Care 2006;10:R106.

12. Papazian L, Doddoli C, Chetaille B, et al. A contributive result of open-lung biopsy improves survival in acute respiratory distress syndrome patients. Crit Care Med 2007;35:755-762.

13. Papazian L, Thomas P, Bregeon F, et al. Open-lung biopsy in patients with acute respiratory distress syndrome. Anesthesiology 1998;88:935-944.

14. Patel SR, Karmpaliotis D, Ayas NT, et al. The role of openlung biopsy in ARDS. Chest 2004;125:197-202.

15. de Hemptinne Q, Remmelink M, Brimioulle S, Salmon I, Vincent JL. ARDS: a clinicopathological confrontation. Chest 2009;135:944-949.

16. Thille AW, Esteban A, Fernandez-Segoviano P, et al. Comparison of the Berlin definition for acute respiratory distress syndrome with autopsy. Am J Respir Crit Care Med 2013;187:761-767.

17. Cardinal-Fernandez P, Bajwa EK, Dominguez-Calvo A, Menendez JM, Papazian L, Thompson BT. The presence of diffuse alveolar damage on open lung biopsy is associated with mortality in patients with acute respiratory distress syndrome: a systematic review and meta-analysis. Chest 2016;149:1155-1164.

18. Lorente JA, Cardinal-Fernandez P, Munoz D, et al. Acute respiratory distress syndrome in patients with and without diffuse alveolar damage: an autopsy study. Intensive Care Med 2015;41:1921-1930.

19. Kao KC, Hu HC, Chang CH, et al. Diffuse alveolar damage associated mortality in selected acute respiratory distress syndrome patients with open lung biopsy. Crit Care 2015;19:228.

20. Ashbaugh DG, Bigelow DB, Petty TL, Levine BE. Acute respiratory distress in adults. Lancet 1967;2:319-323.

21. Phua J, Badia JR, Adhikari NK, et al. Has mortality from acute respiratory distress syndrome decreased over time? A systematic review. Am J Respir Crit Care Med 2009;179:220-227.

22. Bernard GR, Artigas A, Brigham KL, et al. The American-European Consensus Conference on ARDS: definitions, mechanisms, relevant outcomes, and clinical trial coordination. Am J Respir Crit Care Med 1994;149(3 Pt 1):818-824.

23. Katzenstein AL, Bloor CM, Leibow AA. Diffuse alveolar damage: the role of oxygen, shock, and related factors. A review. Am J Pathol 1976;85:209-228.

24. Leaver SK, Evans TW. Acute respiratory distress syndrome. BMJ 2007;335:389-394.

25. Wheeler AP, Bernard GR. Acute lung injury and the acute 
respiratory distress syndrome: a clinical review. Lancet 2007;369:1553-1564.

26. Calfee CS, Delucchi K, Parsons PE, et al. Subphenotypes in acute respiratory distress syndrome: latent class analysis of data from two randomised controlled trials. Lancet Respir Med 2014;2:611-620.

27. Wong AK, Walkey AJ. Open lung biopsy among critically ill, mechanically ventilated patients: a metaanalysis. Ann Am Thorac Soc 2015;12:1226-1230.

28. Libby LJ, Gelbman BD, Altorki NK, Christos PJ, Libby DM. Surgical lung biopsy in adult respiratory distress syndrome: a meta-analysis. Ann Thorac Surg 2014;98:12541260.

29. Brusselaers N, Vogelaers D, Blot S. The rising problem of antimicrobial resistance in the intensive care unit. Ann Intensive Care 2011;1:47.

30. Tabah A, Cotta MO, Garnacho-Montero J, et al. A systematic review of the definitions, determinants, and clinical outcomes of antimicrobial de-escalation in the intensive care unit. Clin Infect Dis 2016;62:1009-1017.

31. Tan SK, Burgener EB, Waggoner JJ, et al. Molecular and culture-based bronchoalveolar lavage fluid testing for the diagnosis of cytomegalovirus pneumonitis. Open Forum Infect Dis 2016;3:ofv212.

32. Meersseman W, Lagrou K, Maertens J, et al. Galactoman- nan in bronchoalveolar lavage fluid: a tool for diagnosing aspergillosis in intensive care unit patients. Am J Respir Crit Care Med 2008;177:27-34.

33. Jain P, Sandur S, Meli Y, Arroliga AC, Stoller JK, Mehta AC. Role of flexible bronchoscopy in immunocompromised patients with lung infiltrates. Chest 2004;125:712-722.

34. Flori P, Bellete B, Durand F, et al. Comparison between real-time PCR, conventional PCR and different staining techniques for diagnosing Pneumocystis jiroveci pneumonia from bronchoalveolar lavage specimens. J Med Microbiol 2004;53(Pt 7):603-607.

35. Churg A, Wright JL, Tazelaar HD. Acute exacerbations of fibrotic interstitial lung disease. Histopathology 2011;58:525-530.

36. Bradley B, Branley HM, Egan JJ, et al. Interstitial lung disease guideline: the British Thoracic Society in collaboration with the Thoracic Society of Australia and New Zealand and the Irish Thoracic Society. Thorax 2008;63 Suppl 5:v1-v58.

37. Richeldi L, du Bois RM, Raghu G, et al. Efficacy and safety of nintedanib in idiopathic pulmonary fibrosis. $\mathrm{N}$ Engl J Med 2014;370:2071-2082.

38. King TE Jr, Bradford WZ, Castro-Bernardini S, et al. A phase 3 trial of pirfenidone in patients with idiopathic pulmonary fibrosis. N Engl J Med 2014;370:2083-2092. 